\title{
EBSD study on YBCO textured bulk samples: correlation between crystal growth and 'microtexture'
}

\author{
D Grossin ${ }^{1}$, C Henrist ${ }^{2}$, J-Ph Mathieu ${ }^{2}, \mathbf{S}$ Meslin $^{1}$, C Harnois ${ }^{1}$, \\ J-G Noudem $^{1}$, R Cloots $^{2}$ and D Chateigner ${ }^{1}$ \\ ${ }^{1}$ CRISMAT Laboratory, ENSICAEN, 6 Bd Maréchal Juin, 14050 Caen Cedex, France \\ ${ }^{2}$ SUPRATECS/CAT $\mu$, University of Liège, Bâtiment B6, Sart-Tilman 4000 Liège, Belgium \\ E-mail: david@grossin.com
}

Received 24 August 2005, in final form 11 November 2005

Published 10 January 2006

Online at stacks.iop.org/SUST/19/190

\begin{abstract}
This work describes an electron backscattered diffraction (EBSD) study of the perovskite-derived structures $\mathrm{YBa}_{2} \mathrm{Cu}_{3} \mathrm{O}_{7-\delta}$. After having pointed out the difficulties of EBSD analyses in resolving the orientations of these pseudo-cubic structures, various $\mathrm{YBaCuO}$ bulk samples are analysed and the correlation between the microstructure, crystal growth and global texture, determined by neutron diffraction, is carried out. Homogeneous 'microtexture' with small subdomain misorientation of $12^{\circ}$ are measured for YBCO top seeding melt textured growth (TSMTG) samples. YBCO perforated samples also exhibit misoriented subdomains, giving rise to a heterogeneous 'microtexture' correlated to the YBCO growth front and to the pattern used for the perforating.
\end{abstract}

(Some figures in this article are in colour only in the electronic version)

\section{Introduction}

The quantitative determination and interpretation of crystallographic textures is of fundamental importance in materials like $\mathrm{YBa}_{2} \mathrm{Cu}_{3} \mathrm{O}_{7-\delta}(\mathrm{Y} 123)$ in which the anisotropic character is aimed to be used in practical applications. The wellestablished $\mathrm{x}$-ray or neutron diffraction quantitative texture analysis (QTA) global techniques can nowadays be complemented by local orientation determinations like microbeam Laue diffraction [1, 2], sometimes called 'microtexture' analyses. Local orientation determinations have received increasing attention with the development of EBSD mounted on scanning electron microscopes (SEMs) [3], because they provide microstructural insights such as grain orientation mapping or misorientation distribution functions. The main drawback of global techniques is the lack of possible correlation of the crystallographic orientation information with the microstructure, but they do however provide other crucial information on microstrain, crystallite sizes, structure, phase fractions, residual strains and so on in their developments called 'combined analysis' [4]. Global techniques provide information on all these parameters in a statistically relevant way in terms of number of probed crystallites, because they analyse large volumes, typically at the cubic centimetre scale for neutrons, and moreover $10^{4}$ times less for $\mathrm{x}$-rays in such materials. Local characterization obtained by EBSD could in principle probe for structure determination, residual stresses and so on but up to now these approaches have not been developed. However, the great advantage of EBSD lies in its capabilities to provide spatial location of the grains coupled [5] with crystal information such as phase, orientation, crystallinity, and grain boundaries.

\section{Experimental details}

\subsection{Classical top seeding melt textured growth (TSMTG) process}

The practical applications of the bulk superconductors $\mathrm{YBa}_{2} \mathrm{Cu}_{3} \mathrm{O}_{7-\delta}$ depend crucially on the quality of the texture in the material and, more precisely, on the grain boundary misorientations [6-8], which are known to influence detrimentally the transport critical current density [9-15]. As a ceramic, Y123 specimens can encounter typical 
difficulties in the sample preparation, like cracking or oxygen understoichiometry, and the achievement of good quality EBSD patterns suited for a correct interpretation is difficult, because surface damage, polishing distortion and charging under observation can occur [16]. Since the analysed depth of the material is of the order of $100 \mathrm{~nm}$, the deposition of a conductive coating, either metallic or graphitic, must be avoided $[17,18]$. The spatial resolution offered by EBSD is lower than $500 \mathrm{~nm}$ and fits well with the requirements of multiphase superconductive ceramic characterization [19]. The angular resolution of EBSD, of the order of $1^{\circ}-2^{\circ}$ [20], allows the quantification of misorientation between subgrains or twins and its correlation with the local microstructure The widely used method of top seeding melt textured growth (TSMTG) [21-23] was applied for YBCO growth of single domains. One single domain is a sample which contains only one single textured zone; in our case only the matrix is textured. A mixture of $\mathrm{Y} 123$ and $\mathrm{Y}_{2} \mathrm{BaCuO}_{5}(\mathrm{Y} 211)$ powders (78:20 weight ratio) with $1 \mathrm{wt} \% \mathrm{CeO}_{2}$ was pressed as cylindrical pellets with $\mathrm{SmBa}_{2} \mathrm{Cu}_{3} \mathrm{O}_{7-\delta}[24,25]$ seeds placed on top. The pellets were melt-textured following the thermal cycle described by Leblond et al [24]. The growth starts at the interface between the pressed pellet and the seed and spreads all over the sample volume to become a quasi-single domain. The microstructure so obtained can be described as a continuous textured matrix made of Y123 phase containing numerous inclusions of Y211 phase.

\subsection{TSMTG process with artificially patterned holes}

The TSMTG process is a suitable way to produce disc-shaped bulk samples for applications such as magnetic bearings or superconducting motors. However, many difficulties remain which are related to the bulk characteristics: important porosity, long and non-homogeneous oxygenation, slow cooling rate in liquid nitrogen. In order to improve thermal as well as gaseous exchanges, a novel technique was developed to increase the exchanging interface $[26,27]$. In such an approach, the so-called artificially patterned holed samples are perforated by several vertical channels in which gas species and thermal fluxes are carried. Samples were prepared from commercial powders. The powder mixture was pelletized uniaxially and then sintered at $920^{\circ} \mathrm{C}$ for $24 \mathrm{~h}$. Regularly spaced $1 \mathrm{~mm}$ diameter holes were drilled into sintered bulk samples before the standard melt process is operated. After the growth step, the samples were submitted to an additional annealing under oxygen flow at $450^{\circ} \mathrm{C}$ for $150 \mathrm{~h}$ in order to obtain good superconducting properties [26, 28].

\subsection{Experimental conditions for EBSD data collecting}

YBCO bulk samples were embedded in a phenolic hot mounted resin loaded with carbon (Polyfast, Struers), processed at $180^{\circ} \mathrm{C}$ under $20 \mathrm{~N}$ pressure for $5 \mathrm{~min}$. This resin presents the advantage of poor edge retraction and good conducting properties due to carbon load. The processing temperature and time are mild enough to avoid damage or structure modification of the superconducting specimen. The bulk sample was placed in the resin so that the face to be polished corresponded to the top surface of the sample, i.e. the $(a, b)$ planes. The polishing method was adapted from standard procedures to reach the surface requirements [29] for EBSD characterization. The resins were mounted on a multisample holder and ground with SiC paper \#220, \#1200 and \#4000 at $150 \mathrm{rpm}$ with an applied pressure of $30 \mathrm{~N}$ per sample and using alcohol-based lubricant (DP-Brown, Struers). Ultrasonication in isopropanol of the sample holder, with the resins kept in place, was used between each step to remove any residue from the surface and from the porosity. Two additional polishing steps with 3 and $1 \mu \mathrm{m}$ diamond spray can be applied if necessary, but if the grinding is driven with extreme care, the surface quality can reach acceptable quality only with $\mathrm{SiC}$ papers. The resin was fixed on the microscope sample holder with carbon tape and tilted at $70^{\circ}$, so that the specimen surface lay at $20^{\circ}$ from the incident electron beam. The study was carried out in a field emission gun-environmental scanning electron microscope (FEG-ESEM) (Philips XL-30) equipped with a TSL camera and the OIM monitoring software. The working distance was $30 \mathrm{~mm}$ and the spot size and diaphragm had to be adjusted to enhance signal/noise ratio. All patterns were generated at an acceleration voltage of $25 \mathrm{kV}$. A background was first collected under the same conditions as the Kikuchi patterns and subsequently subtracted from all data. The scans were collected with a medium resolution with a step according to the size of the scanned area and particles to be analysed, and adjusted to record several thousands points for optimum statistical representation. The orientations are described in Bunge's set of the Euler angles convention [30].

\subsection{Data treatment}

The Kikuchi patterns were automatically indexed by the data collection unit, then analysed in the data analysis software package (TSL Software). YBCO TSMTG samples are multiphase materials made of a $\mathrm{YBa}_{2} \mathrm{Cu}_{3} \mathrm{O}_{7-\delta}$ (Y123) matrix with $\mathrm{Y}_{2} \mathrm{BaCuO}_{5}(\mathrm{Y} 211)$ particles. The superconducting Y123 phase is orthorhombic (Pmmm space group) for small $\delta$ values, while the poorly oxygenated, insulating, structure ( $\delta$ from 0.5 to 1 ) is tetragonal ( $P 4 / \mathrm{mmm}$ space group). The EBSD patterns for both these Y123 stoichiometries are, however, very similar, and the set-up resolution could not probe for the differences; the aim of the work is not to identify the oxygenated regions in the sample. Therefore, a tetragonal crystal structure was used in this work for all Y123 stoichiometry. We have observed that the tetragonal phase provided in the TSL database $(P 4 / \mathrm{mmm}, a=b=3.86 \AA$, $c=11.66 \AA)$ gave better results than a user crystal file built from an orthorhombic unit cell (Pmmm, $a=3.811 \AA$, $b=3.879 \AA, c=11.637 \AA$ ). The Y211 phase is also orthorhombic but with a very distinct unit cell (Pnma, $a=$ $12.18 \AA, b=5.659 \AA, c=7.132 \AA)$. As the Y123 pseudocubic cell of the perovskite has parameters $c / 3=3.88 \AA \sim a$ and $b$, the automated indexation procedure can easily lead to a misattribution of crystal cell orientation by confusing one third of the $c$-axis with the $a$ - and $b$-axes. This artefact results in a frequent misorientation of $90^{\circ}$ from the actual crystal position. Correct and incorrect (artefact) indexed patterns are shown respectively in figures 1 (a) and (b). Three simulated patterns (figure 1(c)) highlight the difficulties the software has in indexing the same experimental pattern. In fact, orientations can be rotated by $90^{\circ}$ around three perpendicular axes without being differentiated by the simulation software. This comes 
(a)

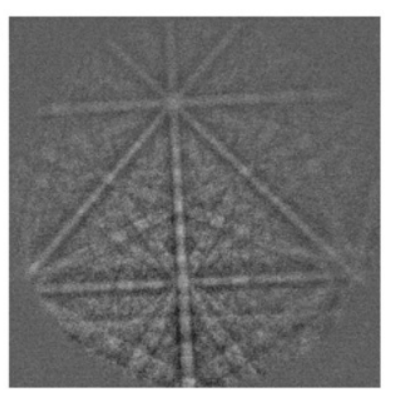

(b)

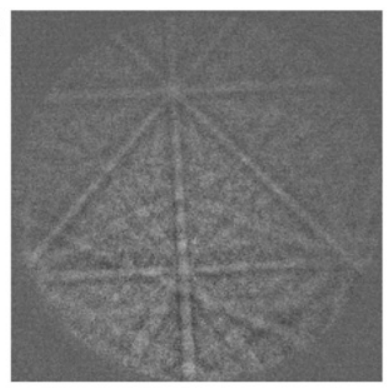

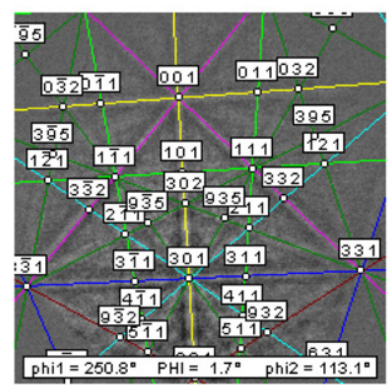

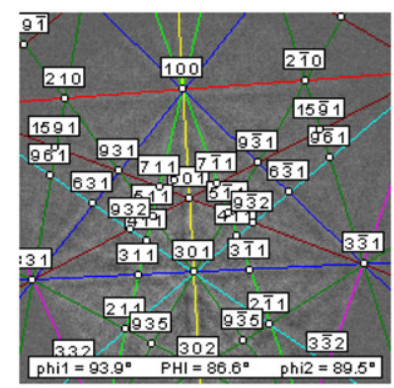

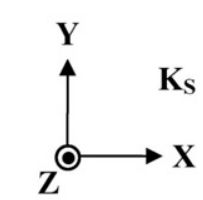
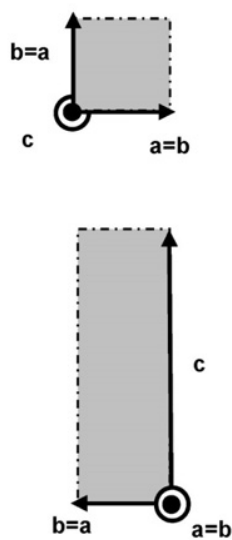

(c)
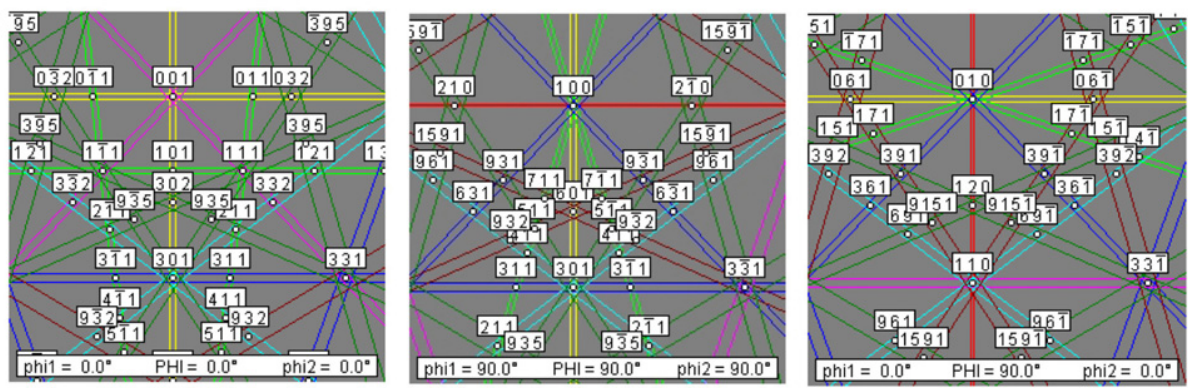

Scan 01

Scan 02

Scan 03

( $1.42 \% 90$-misorientation) ( $1.66 \% 90$-misorientation) (1.59\%90-misorientation)

(d)

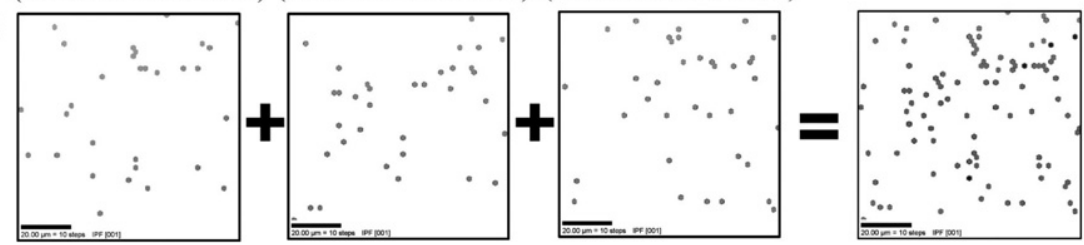

Figure 1. EBSD patterns and indexing. (a) Correct indexing pattern, (b) misindexed pattern. On the right, sketch of the orientation corresponding to the previous patterns, indicating $(a, b, c)$ orientation relative to specimen's orientation $(X, Y, Z)$. (c) Simulated patterns showing the three most frequent solutions for that kind of patterns, two being equivalent by crystal symmetry. (d) Same scan operated three times on the same sample on which black points represent crystallites for which the indexing was the same as in (b), and sum scan illustrating the absence of repeatability of this indexing.

from very comparable band widths and very close locations of the band intersections on the diagram, the two parameters that are used in the indexing step. This was undoubtedly verified on single domains oriented with the $c$-axis normal to the sample surface.

Several scans were measured on the same fixed zone (figure 1(d)) in order to further check the $90^{\circ}$ misorientation origin, which was observed in approximately $1.5 \%$ of the measurements. Two phenomena could explain such misorientations: (i) real material misorientation; (ii) misindexing during the automatic procedure. In the former case, repeating the same measurements on the same sample several times should reveal misoriented crystallites at the same sample locations, while in the latter case such crystallites should be randomly distributed in the sample. Such repeated measurements (figure 1(d)) reveal that the locations of the $90^{\circ}$ misoriented crystallites are different for the different scans. Only $0.19 \%$ of the total number of indexed points were found at the same location on two scans, and not on the third one. None of the misoriented crystallites appeared at the same place for the three scans. We can then attribute these misorientations to automatic misindexing.

A confidence index (CI) is attributed by the software to the proposed pattern indexing. It is related to the difference between the number of votes for the first and the second most probable orientations found by the program. Using such a procedure a $\mathrm{CI}$ of zero indicates that the second proposition has as many chances as the first to be the good one. By imposing 


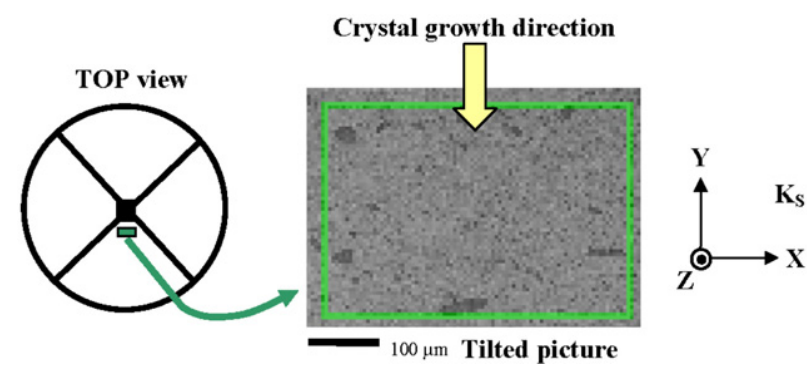

Figure 2. Selected area measured by EBSD on the TSMTG sample, with the corresponding SEM image, and associated sample reference frame $K_{\mathrm{S}}=(X, Y, Z)$.

a severe CI value (i.e. CI $>0.2$ ), the user can efficiently filter points with bad quality patterns or uncertain indexing. Other parameters in the analysis of Kikuchi patterns and their treatment by the Hough transformation procedures [31] can be tuned to enhance the average CI value during the acquisition of a scan. The image quality pattern (IQ) parameter is the sum of the intensity of detected peaks in the Hough transform. A large IQ parameter denotes a high quality of the pattern. At every point, the phase, cell orientation, image quality (IQ) and confidence index (CI) are calculated. Finally, a colour-coded map is calculated according to an inverse pole figure (IPF) plotted for the direction normal to the sample plane to visualize the spatial distribution of crystal orientations. If points with (010) orientations appear like small dots randomly dispersed in a (001) matrix (figure 1(d)), rather than clustered in identified grains, then a filtering of points with a misorientation larger than $80^{\circ}$ relative to the $(001)$ matrix can reasonably be performed without losing the sense.

\section{Results and discussion}

\subsection{TSMTG}

A $350 \mu \mathrm{m} \times 450 \mu \mathrm{m}$ area located at $5 \mathrm{~mm}$ from the seed imprint on top of the sample surface (figure 2) was scanned with a $1 \mu \mathrm{m}$-step hexagonal grid resulting in 157500 patterns. A first map based on the IQ data (figure 3(a)) reveals a contrast related to local variations of surface quality, crystallinity and orientation. Alternating dark and light bands are observed, a priori due to all these effects possibly combined, plus eventually composition variations, which render IQ values from 17 to 115 . Lighter zones are observed which highlight homogeneous zones shaped as grains in the greyish to dark matrix. A phase distribution map (figure 3(b)) confirms the expected global microstructure, which is made of Y123 matrix at $82.1 \%$ in surface proportion with $17.9 \%$ Y 211 inclusions. The homogeneous zones appearing in the IQ image correspond to totally Y211-free areas of the phase map. Individual orientation mapping for each phase gives important information. In the case of the Y211 phase (figure 3(c)), a random orientation is observed (no predominant colour appears). The Y123 phase shows a pronounced orientation, exclusively with [001] directions oriented perpendicular to the analysed surface (figure $3(\mathrm{~d})$ ). This is revealed by a predominant red (mid-grey in print only) colour corresponding to the [001] crystal axis of the inverse pole figure. Small elongated variations of the red (mid-grey in print only) shade (a)

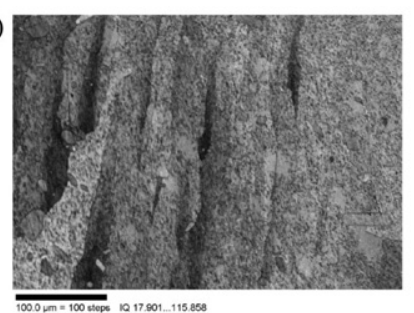

17 115

(b)
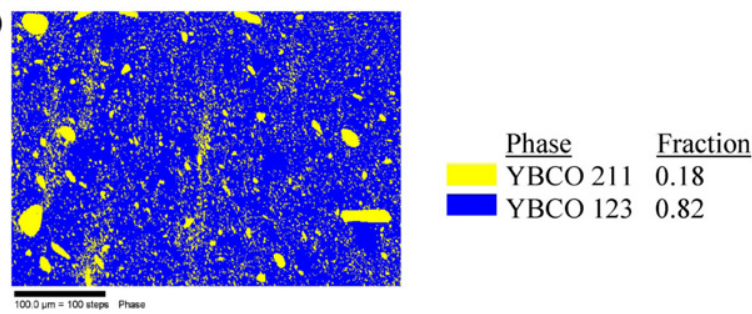

(c)

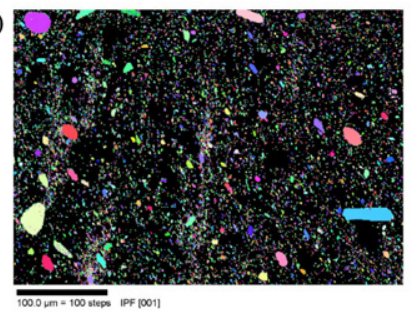

010

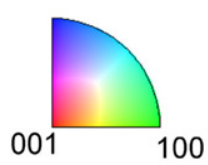

(d)

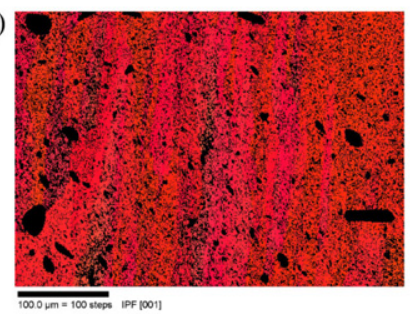

(e)
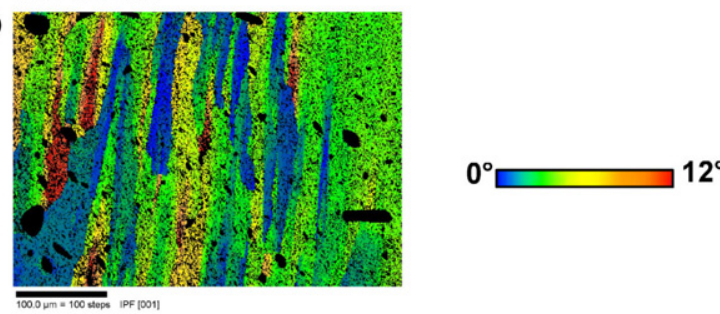

Figure 3. EBSD data on the TSMTG sample. (a) IQ index representation, (b) phase map and relative phase fraction, (c) $001 \mathrm{IPF}$ orientation map for the Y211 phase (black stands for the other phase), (d) 001 IPF orientation map for the Y123 phase, and (e) misorientation map for Y123.

confirm low-angle misorientation between the bands observed in the IQ map.

EBSD allows one to determine small values of misorientation, within a resolution of typically $1^{\circ}$. Using a colour scale from $0^{\circ}$ to $12^{\circ}$, slightly misoriented bands are observed (figure 3(e)) that correspond to subgrains with a mean width comprised between 10 and $100 \mu \mathrm{m}$. The misorientations observed in figure 3 , from one subgrain to another, can be quantified by a line-scan of misorientation (figure 4(a)): discrepancies of more than $5^{\circ}$ are often recorded, and they can reach $15^{\circ}$ for some bands (figures $4(\mathrm{C} 3),(\mathrm{C} 4)$, (C5)). Inside a subgrain, the misorientation from point to point is lower than $2^{\circ}$ (figures $4(\mathrm{C} 1),(\mathrm{C} 2)$ ). Correlation between the crystal growth and the microstructure is highlighted by EBSD 
(a)
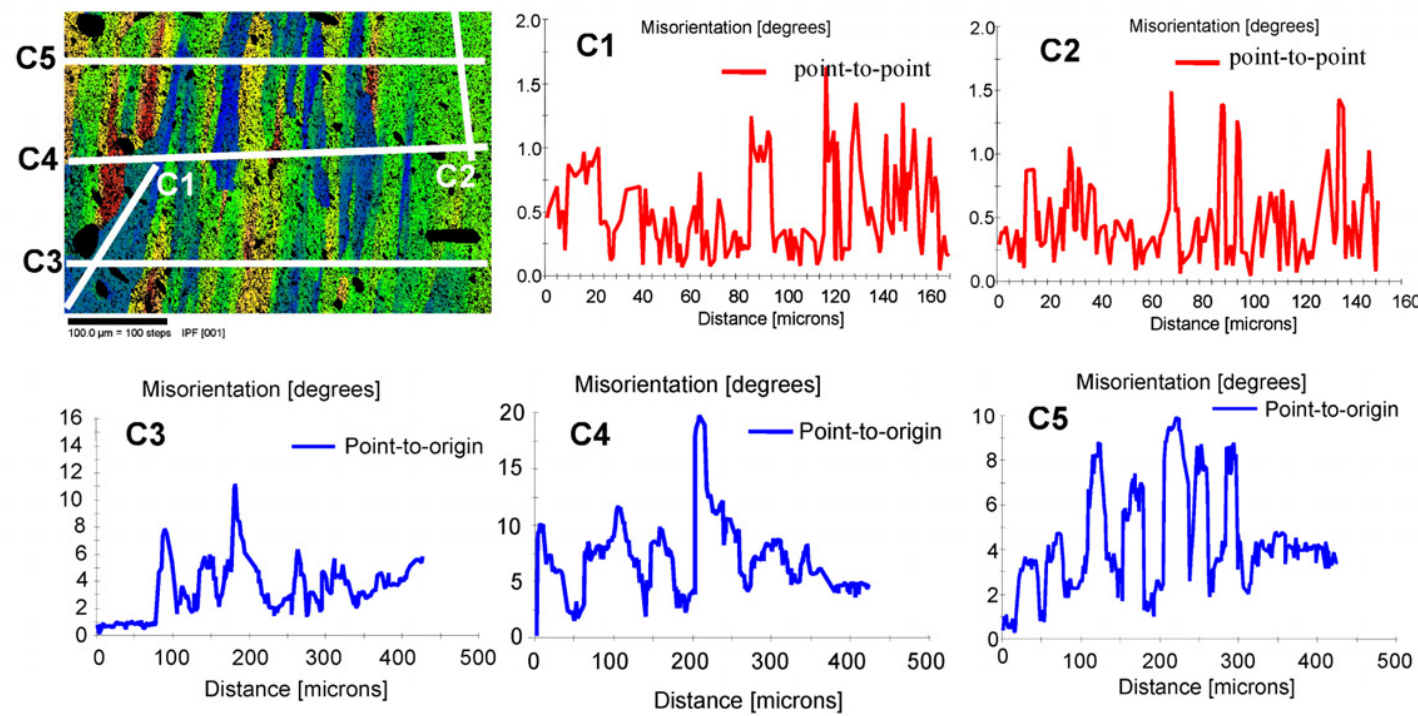

(b)
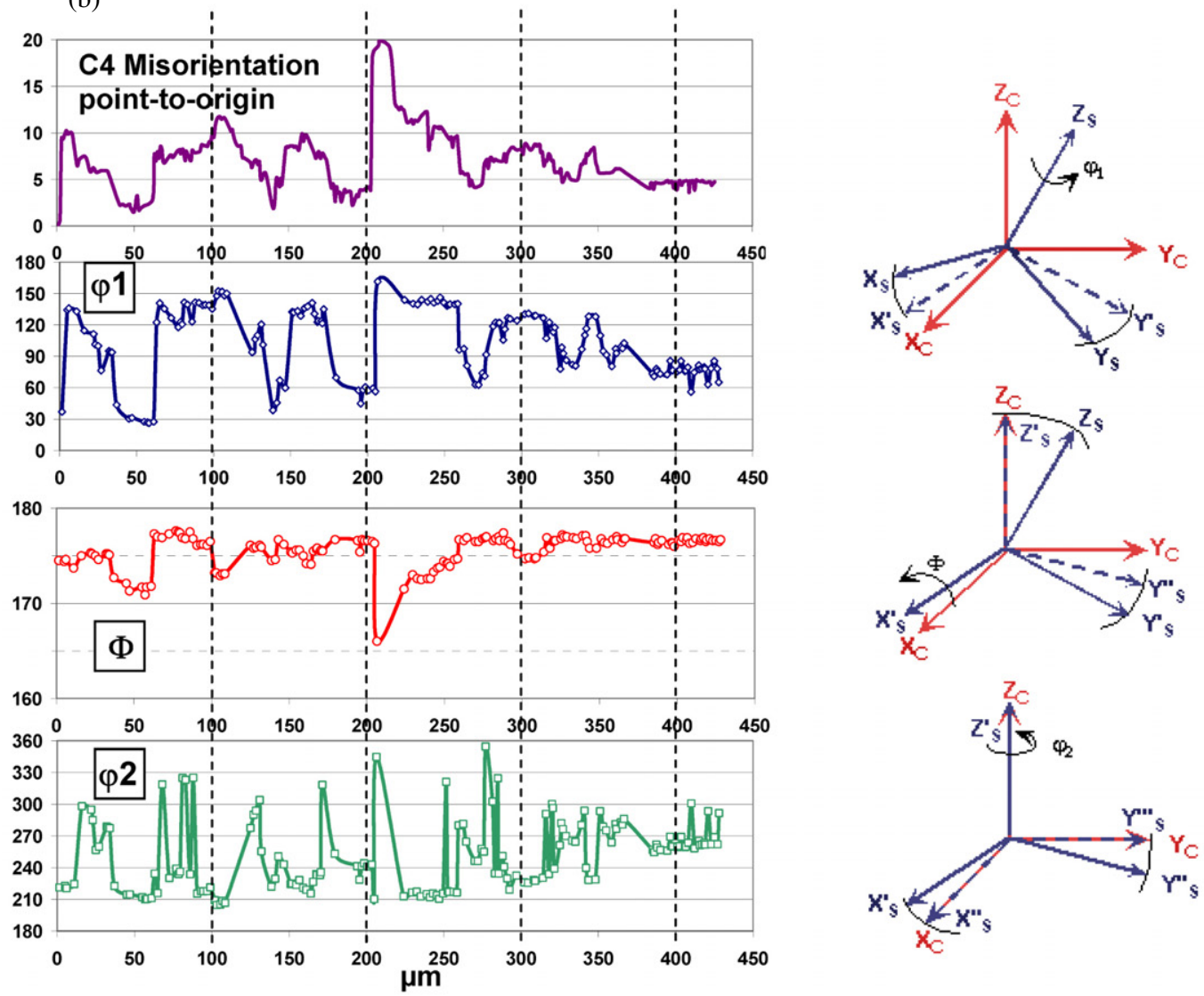

Figure 4. (a) Profile scans ofmisorientations measured on the TSMTG sample for different lines on the sample and (b) corresponding variations of the orientation angles (Bunge's convention) for the line $\mathrm{C} 4$.

measurements: subdomain bands (that are aligned) parallel to each other exhibit elongated shapes with the long axis along the crystal growth direction.

Twist or tilt grain boundaries are peculiar misorientations that occur in such systems, which also delineate subdomains or domains. In order to establish the type of boundary misorientation existing in this sample, the $\mathrm{C} 4$ segment is analysed (figure 4(b)). Using the Euler angle definition of Bunge, the orientation is defined by $g=\left\{\varphi_{1}, \Phi, \varphi_{2}\right\}$, which brings the sample reference frame $Z_{\mathrm{s}}$ into coincidence with the 
(a)

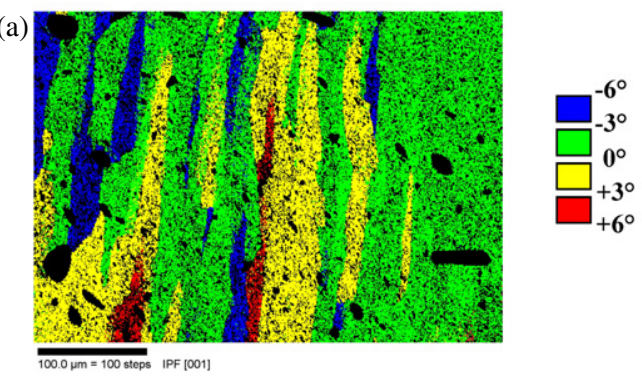

(b)

001

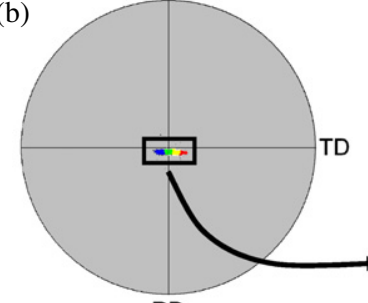

\begin{tabular}{|l|l|}
\hline & $\cdots$ \\
\hline & \\
\hline
\end{tabular}

$\mathrm{RD}$

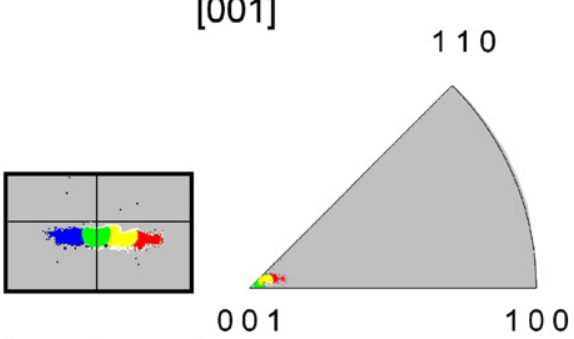

(c)
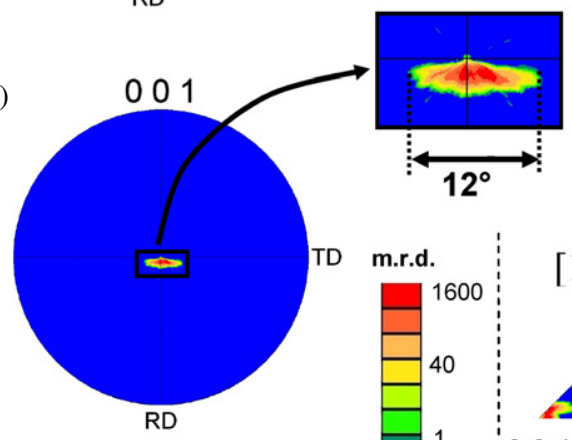

001

100

(d)
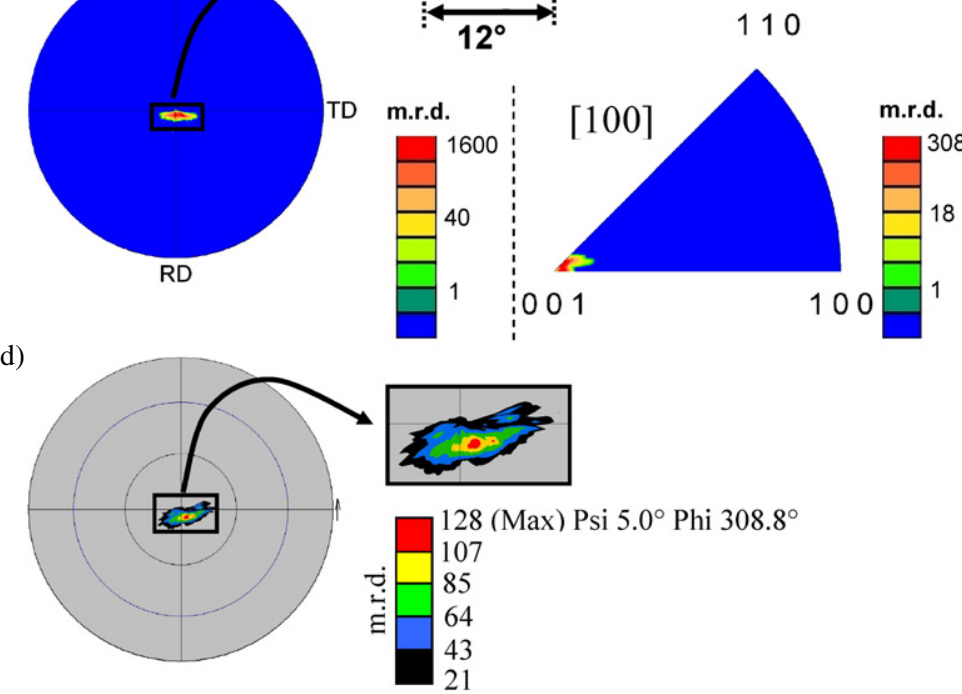

Figure 5. (a) Misorientation map represented as angular variation ranges, calculated from 001 pole figure data, (b) $\{001\}$ pole figure (stereo area projection) and inverse pole figure for the $Z$-direction of the sample represented using the same angular ranges $\left(0^{\circ}\right.$ is for the $Z$-axis), (c) normalized $\{001\}$ pole figure (logarithm density scale, stereo area projection) and inverse pole figure plotted for $Z$, logarithm density scale), (d) experimental x-ray $\{006\}$ pole figure (linear density scale, stereo area projection).

crystal reference frame $Z_{\mathrm{c}}$. The variation of misorientation can be measured along this segment between two measured points by the value $\Delta g=\left\{\Delta \varphi_{1}, \Delta \Phi, \Delta \varphi_{2}\right\}$ (figure 4(b)). We clearly observe that the misorientation fluctuations are associated mainly to the variations of Bunge's angle $\varphi_{1}$. This angle corresponds to the rotation around the normal to the sample plane. In our case the mean $c$-axis direction is parallel to $Z_{\mathrm{s}}$. We can then conclude that $\varphi_{1}$ variations are associated to twist boundaries. This identifies the coloured bands in figure 4(a) to twisted domains around c. Except at a few points, the angle $\Phi$ is only weakly varying. This angle is strongly linked to the tilt boundaries, which consequently are only ranging around $5^{\circ}-10^{\circ}$ at maximum. The average value of $\Phi$ around $175^{\circ}$ (almost $180^{\circ}$ ) is coherent with $c$-axes perpendicular to the sample plane, since normal diffraction does not differentiate $(h k \ell)$ from $(\bar{h} \bar{k} \bar{\ell})$ planes. In this configuration of $\Phi$ values, we observe $\varphi_{2}$ values at either $0^{\circ}, 90^{\circ}, 180^{\circ}$ or $270^{\circ}$ from $\varphi_{1}$. This is coherent with a strong in-plane orientation of the a and $\mathbf{b}$ axes since for $\Phi=180^{\circ}$ (or $0^{\circ}$ ) the $\varphi_{2}$ rotation also represents a rotation around the normal to the sample plane. The overall tilt misorientations are of lower magnitude than the twist misorientations, and never exceed $10^{\circ}$. Furthermore, the misorientations detected as coloured bands in figure 3(e) are often not linked to tilted domains. For instance the largest tilt event occurring on the $\mathrm{C} 4$ line (around step 200 in $\Phi$ variation) is clearly detected as misoriented in the misorientation image, but at larger steps along the same line, a lot of misorientations are observed for a constant $\Phi$ value (without tilt variations). 


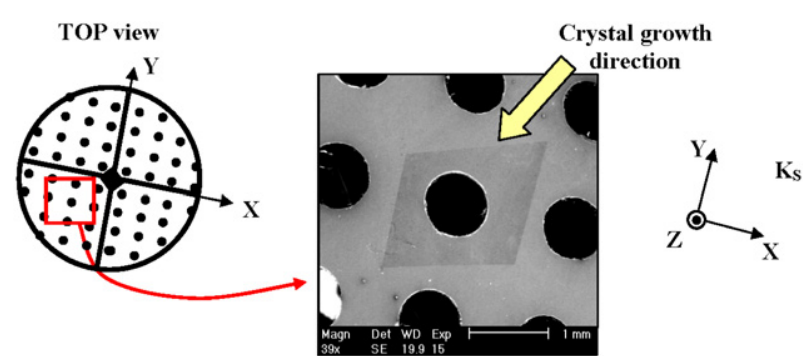

Figure 6. Selected area measured by EBSD on artificially patterned holed TSMTG sample, and corresponding sample reference frame.

The orientation fluctuations in subdomains can also be visualized with the help of pole figures (PFs). Relative to an arbitrary $0^{\circ}$ misorientation corresponding to $Z$, the spacedistribution of the misorientations in the sample (figure 5(a)) can be partitioned into four $3^{\circ}$ intervals. On this figure one can see that at this surface scale most of the grains are misoriented by around $\pm 3^{\circ}$, and some up to $\pm 6^{\circ}$. The $\{001\}$ pole figure (figure 5(b)) shows one elongated spot in the centre, corresponding to a tendency for the $c$-axes to incline towards $\pm X$. The combination of figures 5(a) and (b) indicates there is no correlation between the location of the crystallites in the material and their misorientation relative to $Z$.

The full quantitative texture analysis from the EBSD measurements was operated through the calculation of the orientation distribution function (ODF) by discrete binning $\left(1^{\circ}\right)$ [32]. The normalized EBSD $\{001\}$ pole figure recalculated from the ODF (figure 5(c)) shows a unique peak spread over $12^{\circ}$ in the $X$ direction and $5^{\circ}$ along $Y$, at the 1 m.r.d. level. The corresponding maximum peak density is approximately 1600 m.r.d. (multiple of a random distribution). This is a very high degree of crystallite orientation (a sample without any preferred orientation would exhibit normalized pole figures with 1 m.r.d. values everywhere). The inverse pole figure plotted for the $Z$-axis of the sample shows that only $c$-axes are close to $Z$, in accordance with the $\{001\}$ pole figure. However, such high density levels must be interpreted with care. The analysed surface represents only around $0.2 \mathrm{~mm}^{2}$, and electrons are only backscattered from the very near surface. This corresponds to a volume far from representing the whole sample volume. An analysis operated on larger sample volumes, as accessible using neutron or x-ray measurements, would provide a better statistical reliability. For comparison, the normalized $\mathrm{x}$-ray $\{006\}$ pole figure is shown in figure 5(d) (normalized by direct intensity integration [4]). The analysed surface is approximately twenty times higher than the one analysed by EBSD diffraction. The volume concerned is larger, around $10 \mathrm{~mm}^{2} \times 10 \mu \mathrm{m}$, and the pole figure has been measured using a $2.5^{\circ} \times 2.5^{\circ}$ grid resolution. The same pole anisotropy is observed using $\mathrm{x}$-rays, with however a larger extension which decreases the maximum orientation density accordingly. Neutron diffraction will be done on this sample in order to analyse the misorientation on volume.

\subsection{Artificially patterned holed TSMTG}

Texture studies on an artificially patterned TSMTG sample have already been carried out using $\mathrm{x}$-ray and neutron (a)

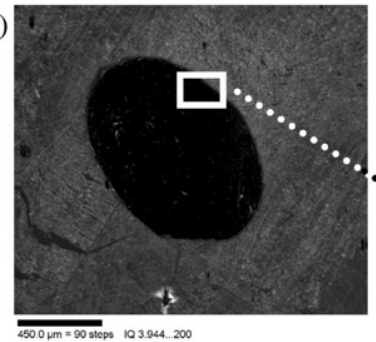

4
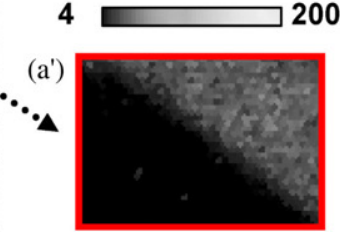

(b)

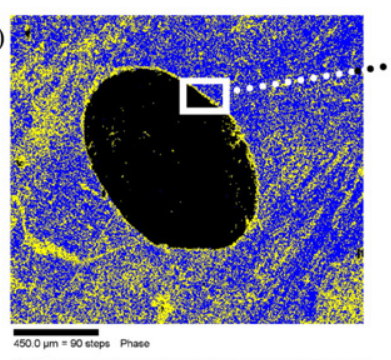

(b')

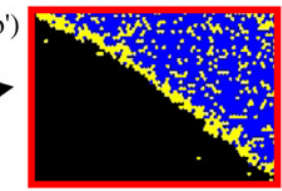

Phase

Fraction

YBCO 1230.58

YBCO $211 \quad 0.42$

(c)
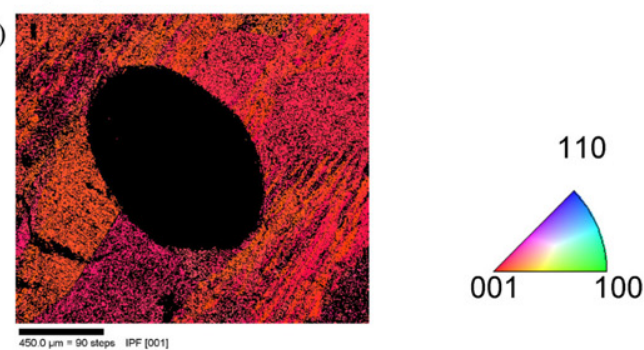

(d)
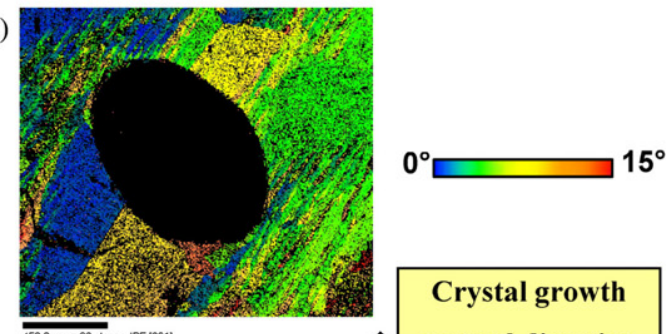

(e)

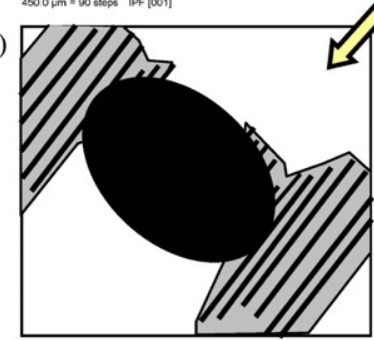

\section{Crystal growth} normal direction

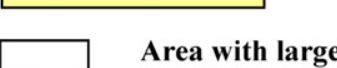

subdomains were observed

Area with small

subdomains were observed

Small subdomain

Figure 7. EBSD data on artificially patterned holed TSMTG sample (a) IQ index representation, $\left(a^{\prime}\right)$ IQ index representation zoom, (b) phase map and relative phase fraction, $\left(\mathrm{b}^{\prime}\right)$ phase map and relative phase fraction zoom, (c) 001 IPF orientation map for the Y123 phase, (d) misorientation map for the Y123 phase, and (e) schema of microstructure/microtexture.

diffraction experiments [26]. Those measurements have shown that the texture development in the total volume, as seen macroscopically, is not influenced by the presence of holes in the pellet [26]. At the same time, SEM microscopic observations indicate that the local microstructure is preserved even in the vicinity of holes. However, EBSD is a technique able to investigate the local texture around or between 
(a)

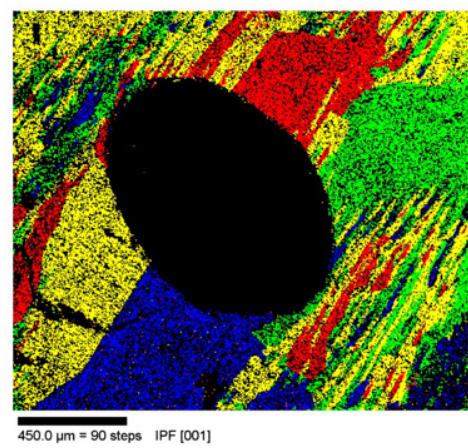

(b)

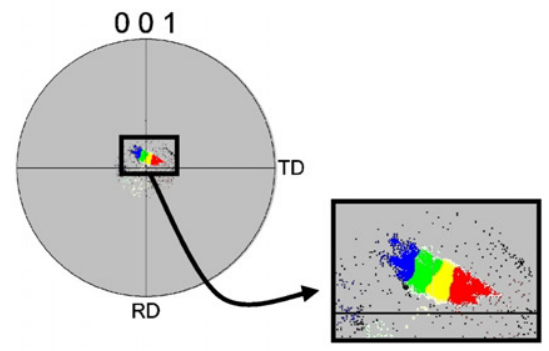

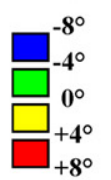

[001]

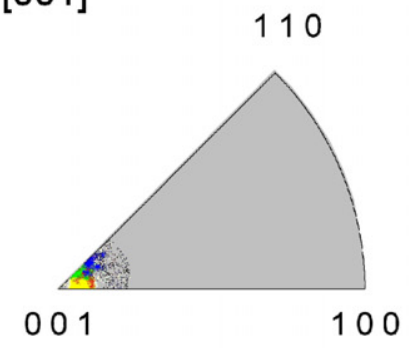

(c)
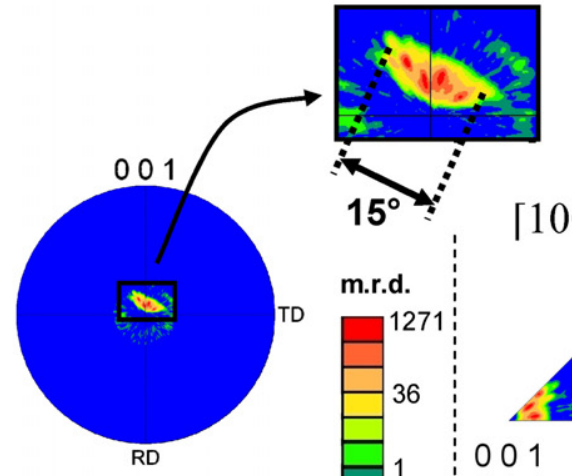

100

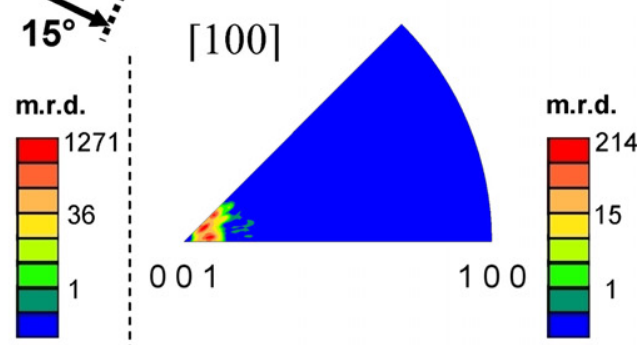

(d)

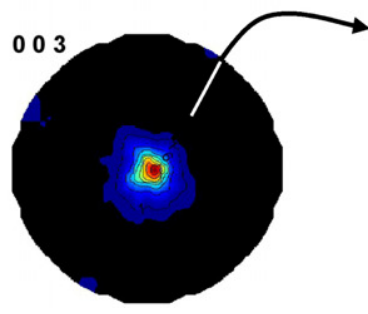

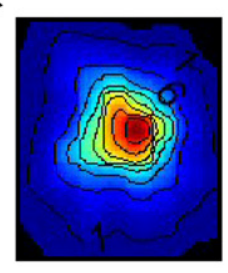

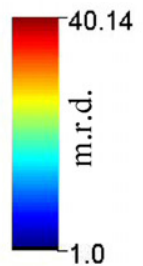

Figure 8. (a) Misorientation map calculated from 001 pole figure data; (b) unnormalized (001) pole figure (stereo area projection) and [001] inverse pole figure; (c) calculated (001) pole figure (logarithm density scale, stereo area projection) and calculated [001] inverse pole figure (logarithm density scale) and (d) calculated neutron (003) pole figure (logarithm density scale, stereo area projection).

the holes, in order to detect eventually more local texture perturbations introduced by hole patterning. The sample characterization (figure 6) was performed following the same procedure as for the classical TSMTG pellets. We present here a detailed study of the zone located around one hole (figure 7).

Image quality mapping of the sample (figure 7(a)) reveals that low IQ indices are located on a $25 \mu \mathrm{m}$ thick zone around the hole and cracks. The associated EBSD patterns shown for the matrix in this region clearly show the worse quality of the near-hole diagram. Interestingly, the associated phase distribution map (figure 7(b)) suggests a high concentration of
Y211 inclusions near the holes and cracks. This could result from a worse image quality as an overall feature for Y211 patterns. However, the IQ levels are typically the same for both Y211 and Y123 phases, and EDX analyses under SEM could not evidence composition variations near holes. But areas close to holes (or cracks) also are less planar and regular. Since EBSD is very sensitive to surface perfection, this confusing indexing is attributed to it.

The Y123 growth remains strongly textured, as denoted by the inverse pole figure map calculated for the direction of hole axes ( $Z$-direction, figure $7(\mathrm{c})$ ). But the orientation 
(a)
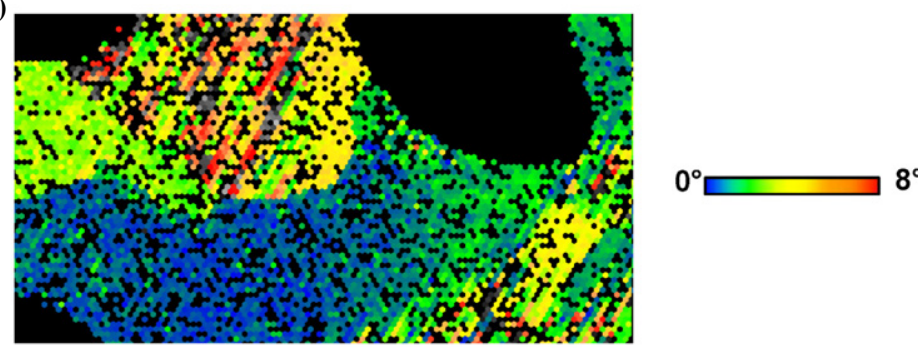

$500.0 \mathrm{\mu m}=25$ steps
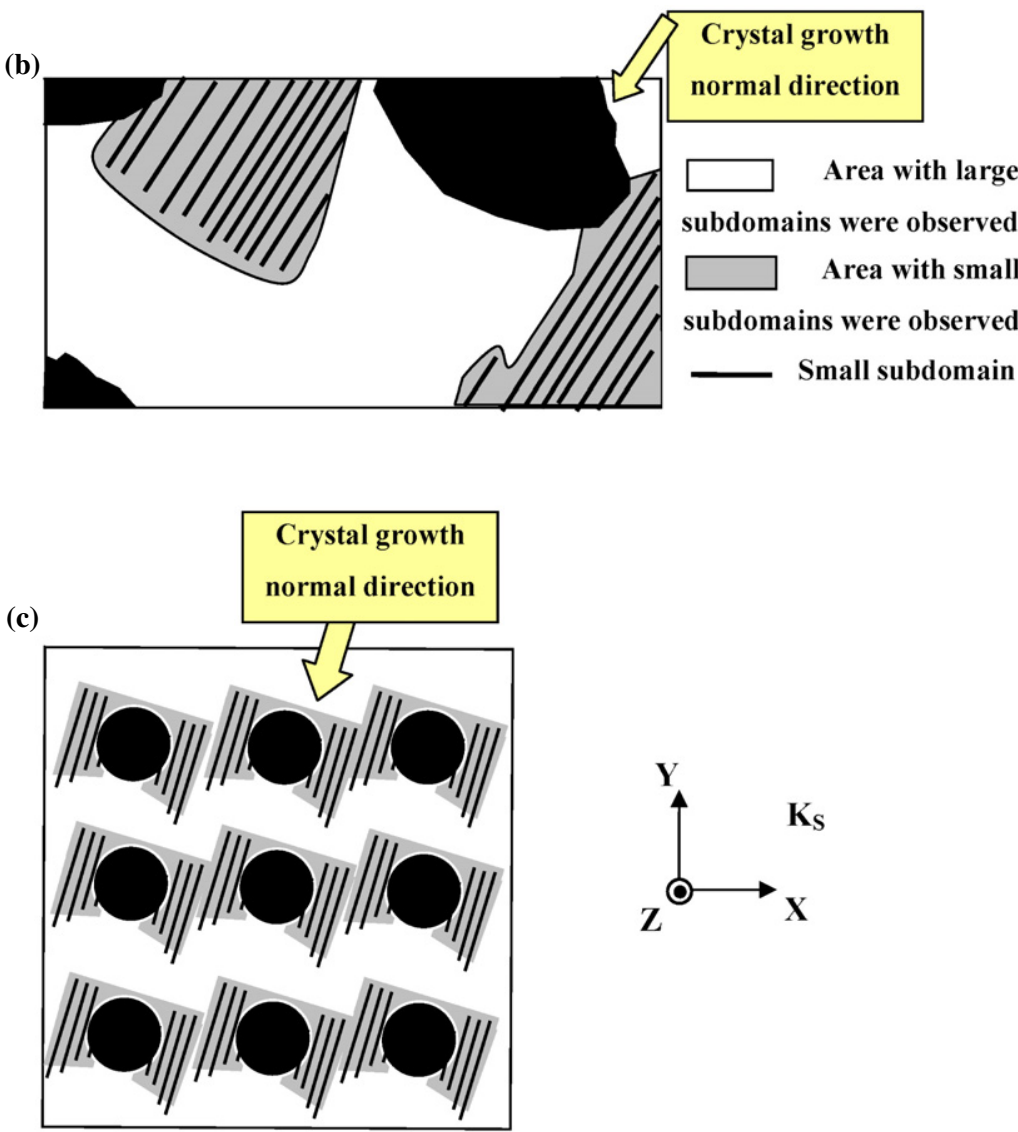

Figure 9. EBSD data and schema on other selected area on artificially patterned holed TSMTG sample. (a) Misorientation map for the Y123 phase, and (b) schema of microstructure/microtexture. (c) Multi-hole schema of microstructure/microtexture.

map exhibits two kinds of zones, depending on the extent of orientation domains (figure 7(d)): small 40-100 $\mu$ m wide subdomains, alternating along the diagonal to the image, and large subdomains, 300-500 $\mu \mathrm{m}$ wide, in the perpendicular direction (see figure 7(e) for a schematic drawing). All these subdomains are elongated in shape and are respectively oriented parallel between them, and perpendicular to the YBCO growth front (recrystallization front) that shift from the top centre (seed) of the pellets to the periphery of the pellets by sympathetic nucleation.

In order to highlight the subdomain orientation variations, EBSD data were discretized as previously in four $4^{\circ}$ domains of misorientation. The [001] inverse pole figure map (figure $8(\mathrm{a})$ ) reveals alternating bands of misorientations up to $\pm 8^{\circ}$. The orientation transitions between subdomains are uniformly distributed, and range between $4^{\circ}$ and $12^{\circ}$ whatever their sizes.
The EBSD normalized $\{001\}$ pole figure (figure 8(c)) shows as previously an elongated peak, with a peak width of $15^{\circ}$ at 1 m.r.d. level. The maximum density around 1300 m.r.d. is coherent with a larger pole dispersion in this sample compared to the previous one.

Neutron texture analysis was processed on this sample in order to probe for the volume texture at the $\mathrm{cm}^{3}$ scale [4]. Figure 8(d) shows the (003) pole figure recalculated from the ODF refined in the Maud software [33, 34] using the E-WIMV model [35] and the combined analysis [4]. One pole with a maximum density of 40 m.r.d. is observed, with a consequently larger dispersion. This effect is typically explained by the considerably larger crystallite number analysed in the case of neutron scattering compared to EBSD, rendering a better statistic in such samples.

EBSD analysis is illustrated on another typical area of the same sample on figure 9. The misorientation map of the Y123 
phase (figures 9(a) and (b)) shows the same textural patterns in terms of misorientations. The misorientation distribution is shown schematically for the whole sample in figure 9 (c). The sample perforation results in an inhomogeneous local texture, again with subdomain variations, but at the global scale of the sample surface the texture resembles a homogeneous pattern in which subdomains are regularly distributed and patterned in a fashion that follows the perforated hole.

\section{Conclusion}

EBSD analyses on two $\mathrm{YBaCuO}$ bulk samples elaborated by TSMTG, one being perforated before melting, allow us to correlate the microstructure and local texture variations. Homogenous local texture with small subdomain misorientations up to $12^{\circ}$ are observed and quantified for the non-perforated YBaCuO TSMTG sample. Subdomains could be highlighted, and the misorientation quantification opens the way to misorientation optimization. In contrast, local texture variations are observed in the case of the $\mathrm{YBaCuO}$ perforated TSMTG sample, which are linked to a specific microstructure relative to the $\mathrm{YBaCuO}$ growth front. The $\mathrm{Y} 123$ misorientations are of the same order of magnitude, up to $15^{\circ}$ in this case. But the local texture and microstructure of this sample is heterogeneous and guided by the distribution of patterned holes.

\section{Acknowledgments}

D Grossin and S Meslin are grateful to the French Ministry of Research and Technology for their PhD fellowships. J-Ph Mathieu thanks FRIA (Fonds pour la Formation a la Recherche dans l'Industrie et dans l'Agriculture, Brussels) for financial support of his doctorate thesis. Professor D Chateigner greatly acknowledges the Région basse-normandie for its partial financing of the $\mathrm{x}$-ray measurement set-up. The authors also thank the European Supermachines Network (HPRN-CT2000-0036) for financial support and their members for several comments. Part of this work results from research activities in the 'EFFORT' network.

\section{References}

[1] Wenk H R, Heidelbach F, Chateigner D and Zontone F 1997 J. Synchrotron Radiat. 495

[2] Adams B L, Wright S I and Kunze K 1993 Metall. Trans. A 24 819

[3] Dingley D 1999 J. Microsc. 195170

[4] Chateigner D 2004 Combined Analysis, web link http://www. ecole.ensicaen.fr/ $\sim$ chateign/texture/combined.pdf

[5] Randle V and Engler O 2000 Introduction to Texture Analysis (London: Gordon and Breach Science Publishers)
[6] Diko P 1995 Phys. Rev. B 5213658

[7] Diko P, Todt V R, Miller D J and Goretta K C 1997 Physica C 278192

[8] Ogasawara K, Sakai N and Murakami M 2000 Supercond. Sci. Technol. 13688

[9] Sandiumenge F, Martınez B and Obradors X 1997 Supercond. Sci. Technol. 10 A93

[10] Sandiumenge F, Vilalta N, Rabier J and Obradors X 2001 Phys. Rev. B 64184515

[11] Dimos D, Chaudhari P, Mannhart J and Leoues F K 1988 Phys. Rev. Lett. 61219

[12] Dimos D, Chaudhari P and Mannhart J 1990 Phys. Rev. B 414038

[13] Verebelyi D T et al 2000 Appl. Phys. Lett. 761755

[14] Feldmann D M et al 2001 Appl. Phys. Lett. 793998

[15] Pernet M, Chateigner D, Germi P, Dubourdieu C, Thomas O, Sénateur J P, Chambonnet D and Belouet C 1994 Physica C 235-240 627

[16] Goldstein J I, Newbury D E, Echlin P, Joy D C, Romig A D J, Lyman C E, Fiori C and Lifshin E 1992 Scanning Electron Microscopy and X-ray Microanalysis 2nd edn (New York: Plenum)

[17] Schwartz A J, Kuma M and Adams B L 2000 Electron Backscattered Diffraction in Materials Science (New York: Kluwer-Academic)

[18] Koblischka-Veneva A and Mücklich F 2002 Cryst. Eng. 5235

[19] Koblischka-Veneva A, Koblischka M R, Simon P, Ogasawura K and Murakami M 2003 Physica C 392-396 601

[20] Prior D J 1999 J. Microsc. 195217

[21] Morita M, Takebayashi S, Tanaka M, Kimura K, Myamoto K and Sawano K 1991 Adv. Supercond. 3733

[22] Mathieu J-Ph, Garcia Cano I, Koutzarova T, Rulmont A, Vanderbemden Ph, Dew-Hughes D, Ausloos M and Cloots R 2004 Supercond. Sci. Technol. 17169

[23] Cloots R, Koutzarova T, Mathieu J-Ph and Ausloos M 2005 Supercond. Sci. Technol. 18 R9

[24] Leblond C, Monot I, Provost J and Desgardin G 1999 Physica C 311211

[25] Gautier-Picard P 1998 PhD Thesis University of Grenoble

[26] Noudem J-G, Meslin S, Harnois C, Chateigner D and Chaud X 2004 Supercond. Sci. Technol. 17931

[27] Tournier R, Chaud X, Isfort D and Shi D 2003 European Patent Pending 033354011.3

[28] Leblond C, Monot I, Bourgault D and Desgardin G 1999 Supercond. Sci. Technol. 12405

[29] Katrakova D, Damgaard M J and Mücklich F 2001 Structure 38 20 (Published by Struers)

[30] Bunge H-J 1982 Texture Analysis in Materials Science (London: Butterworths) (1993 Reprint: Göttingen: Cuvillier)

[31] TSL: Orientation Imaging Microscopy software user manual, edition 2002, TexSEM Lab Inc., Draper, Utah

[32] Matthies S and Vinel G W 1994 Proc. ICOTOM-10; Mater. Sci. Forum 157-162 1641

[33] Guilmeau E, Lambert S, Chateigner D, Noudem J-G and Ouladiaf B 2003 Mater. Sci. Eng. B 104107

[34] Lutterotti L, Matthies S and Wenk H R 1999 Textures of Materials vol 2, ed J A Szpunar (Ottawa: NRC Research Press) p 1599

[35] Morales M, Chateigner D, Lutterotti L and Ricote J 2002 Mater. Sci. Forum 408-412 113 